\title{
Acute Tryptophan Depletion and Functional Brain Imaging in Irritable Bowel Syndrome
} (Gut 2011;60:1196-1203)

\section{Hee Man Kim}

Division of Gastroenterology, Department of Internal Medicine, Myongji Hospital, Kwandong University College of Medicine, Goyang, Gyeonggi-do, Korea

\section{Summary}

Serotonin (5-hydroxytryptamine; 5-HT) is an important signaling molecule in the brain-gut axis, and abnormalities of 5-HT signaling system in the gastrointestinal (GI) tract have been investigated in irritable bowel syndrome (IBS). ${ }^{1}$ However, there are few studies on of acute tryptophan depletion (ATD)-induced 5-HT modulation at the brain level in IBS.

The current study by Labus et $\mathrm{al}^{2}$ is worth examining because it provides novel information on brain network and the augmented activity that may play an important role in pathophysiology of irritable bowel syndrome. This study was aimed at testing 2 hypotheses. The first was that ATD in healthy women increases the engagement of an emotional arousal circuit during rectal distension. The second was that these changes in the brain are consistent with those changes in female patients with constipation predominant IBS (IBS-C). These 2 hypotheses were tested by ATD and functional magnetic resonance image (fMRI) during visceral stimuli.

The subjects were 12 healthy women and 14 female patients with IBS-C. ATD was used as a technique for lowering brain 5-HT. Functional MRI was used as a non-invasive tool to meas- ure brain responses to visceral stimuli. Balloon inflation was used for rectal distension with 2 levels of pressure, including non-painful distension pressure and maximum tolerable pressure. Effective connectivity analysis was used as a mean to test hypotheses regarding neural circuits and network functioning.

In healthy women, balloon inflation in the rectum showed changes in brain activity, and ATD enhanced these changes in brain activity. Brain regions activated with ATD and balloon inflation were the homeostatic afferent network and the emotional arousal network. The amygdala, a key brain region in the emotional arousal network, and the thalamus showed a transient high activation during ATD, while other regions showed sustained activity. Effective connectivity analyses revealed that ATD made a stronger engagement of the emotional arousal circuitry compared to placebo in healthy women during balloon inflation. In other words, ATD resulted in reduced feedback inhibition of the amygdala during an aversive visceral stimulus in healthy women. A similar pattern of effective connectivity within the emotional arousal network was observed in IBS-C patients during rectal distension even without ATD.

Received: December 5, 2011 Revised: December 25, 2011 Accepted: December 27, 2011

(c) This is an Open Access article distributed under the terms of the Creative Commons Attribution Non-Commercial License (http://creativecommons. org/licenses/by-nc/3.0) which permits unrestricted non-commercial use, distribution, and reproduction in any medium, provided the original work is properly cited.

*Correspondence: Hee Man Kim, MD

Department of Internal Medicine, Myongji Hospital, Kwandong University College of Medicine, 697-24 Hwajeong-dong,

Deokyang-gu, Goyang, Gyeonggi-do 412-270, Korea

Financial support: None.

Tel: +82-31-810-6579, Fax: +82-31-969-9500, E-mail: eastin@kd.ac.kr

Conflicts of interest: None. 


\section{Comment}

In the GI tract, 5-HT serves as both a paracrine molecule and a neurotransmitter, and 5-HT signaling is physiologically involved in secretion, motility and perception..$^{3,4}$ Alteration of the 5-HT signaling system in the brain-gut axis has been found to be associated with functional gastrointestinal disorders (FGIDs) including IBS; drugs involved in 5-HT signaling modulation have been clinically and widely used in patients with FGIDs.

Only $5 \%$ of the total $5-\mathrm{HT}$ is found in the brain, whereas $95 \%$ is found in the GI tract. ${ }^{5}$ In the brain, 5 -HT system helps to maintain the normal mental stability, and the alteration of this system is closely associated with mood disorders. The serotonergic neurons in the brain are located in the nuclei of the median raphe. The descending projections of raphe nuclei are involved in the central modulation of pain, whereas the ascending projections are involved in the regulation of mood and cognition. ${ }^{3,6}$ These neurons are involved in the brain-gut axis. Brain 5-HT signaling system has been more extensively investigated in neuropsychiatric disorders such as depression and anxiety than in FGIDs.

There are several studies about an association between altered 5-HT signaling in the brain by ATD and functional gastrointestinal disorders, with varying results. ${ }^{3,78}$ ATD lowers the perceptional threshold of the rectal distension in IBS patients and controls. ${ }^{3}$ ATD causes a significant delayed gastric emptying. On the contrary, another study reported that ATD did not affect GI symptoms in the controls. ${ }^{8}$ This study used VAS scales for scoring GI symptoms, and did not include rectal distension. ${ }^{8}$ The different experimental conditions may affect the different results. The current study arouses interest because ATD-induced alteration of 5-HT in the brain can be partly involved in the pathogenesis of IBS. Additionally, this study introduced 2 unique methods of ATD and fMRI to the field of gastroenterology.

ATD is a dietary technique to lower brain 5-HT and to investigate the role of 5-HT in the pathogenesis of neuropsychiatric diseases. ${ }^{9}$ Tryptophan is the precursor of 5-HT and is an essential amino acid in the diet. In 1974, the administration of a mixture of essential amino acids free of tryptophan was reported to reduce levels of brain tryptophan and 5-HT in the rats. ${ }^{10}$ In 1977, the first use of the tryptophan-free amino acid mixture in human subjects was reported. ${ }^{11}$ The tryptophan-free diet also produced a decline in cerebrospinal fluid tryptophan and 5-hydroxyindolacetic acid in healthy human subjects. ${ }^{12}$ Several studies of FGIDs have used this dietary method. ATD produces sig- nificantly delayed gastric emptying of a solid meal in healthy volunteers. ${ }^{7}$ ATD affects visceral perception in diarrhea predominant IBS and healthy controls. ${ }^{3}$ ATD induces changes in GI and anxiety symptoms in IBS patients. ${ }^{8}$ The current study found that ATD reduced inhibition of an emotional arousal network during aversive visceral stimulation and increased effective connectivity within an emotional arousal network. ${ }^{2}$ In the future, ATD will be broadly used to investigate pathophysiology of FGIDs including IBS.

Functional brain imaging, such as fMRI, provides a noninvasive measurement to indicate the central response to stimuli and a tool for identifying functional brain networks. ${ }^{13}$ In FGID, 2 functional networks are recognized, the homeostatic afferent network and emotional arousal network. ${ }^{13}$ The emotional arousal network is composed of the amygdala and anterior cingulated cortical sub-regions. ${ }^{14}$ The fMRI findings of the current study are consistent with those of previous studies in healthy volunteers, and also newly revealed effective connectivity within an emotional arousal network in IBS-C patients. ${ }^{2}$

Although the current study will contribute to elucidating the role of 5-HT in the brain of patients with IBS-C, the small sample size is a limitation. Another limitation is that ATD can cause a decrease in peripheral 5-HT, which can affect 5-HT signaling in the GI tract. ATD cannot separate central and peripheral 5-HT alteration, and changes in the brain may be affected by the altered peripheral 5-HT signaling.

In conclusion, the current study introduces new research techniques, ATD and fMRI, for investigating FGIDs, and demonstrates that alteration on 5-HT signaling in the brain may play an important role in IBS pathophysiology.

\section{References}

1. Crowell MD, Wessinger SB. 5-HT and the brain-gut axis: opportunities for pharmacologic intervention. Expert Opin Investig Drugs 2007;16:761-765.

2. Labus JS, Mayer EA, Jarcho J, et al. Acute tryptophan depletion alters the effective connectivity of emotional arousal circuitry during visceral stimuli in healthy women. Gut 2011;60:1196-1203.

3. Kilkens TO, Honig A, van Nieuwenhoven MA, Riedel WJ, Brummer RJ. Acute tryptophan depletion affects brain-gut responses in irritable bowel syndrome patients and controls. Gut 2004;53:1794-1800.

4. Camilleri M. Serotonergic modulation of visceral sensation: lower gut. Gut 2002;51(suppl 1):81-86.

5. Sikander A, Rana SV, Prasad KK. Role of serotonin in gastrointestinal motility and irritable bowel syndrome. Clin Chim Acta 2009;403:47-55.

6. Gershon MD, Tack J. The serotonin signaling system: from basic 
understanding to drug development for functional GI disorders. Gastroenterology 2007;132:397-414.

7. Van Nieuwenhoven MA, Valks SD, Sobczak S, Riedel WJ, Brummer RJ. Acute tryptophan depletion slows gastric emptying in females. $\mathrm{Br}$ J Nutr 2004;91:351-355.

8. Shufflebotham J, Hood S, Hendry J, et al. Acute tryptophan depletion alters gastrointestinal and anxiety symptoms in irritable bowel syndrome. Am J Gastroenterol 2006;101:2582-2587.

9. Biggio G, Fadda F, Fanni P, Tagliamonte A, Gessa GL. Rapid depletion of serum tryptophan, brain tryptophan, serotonin and 5-hydroxyindoleacetic acid by a tryptophan-free diet. Life Sci 1974;14: 1321-1329.

10. Gessa GL, Biggio G, Fadda F, Corsini GU, Tagliamonte A. Effect of the oral administration of tryptophan-free amino acid mixtures on serum tryptophan and serotonin metabolism. J Neurochem 1974;22:
869-870.

11. Concu A, Fadda F, Blanco S, Congia S, Lostia M. Mental changes induced by the oral administration of tryptophan-free amino acid mixture in man. IRCS Med Sci 1977;5:520.

12. Williams WA, Shoaf SE, Hommer D, Rawlings R, Linnoila M. Effects of acute tryptophan depletion on plasma and cerebrospinal fluid tryptophan and 5-hydroxyindoleacetic acid in normal volunteers. J Neurochem 1999;72:1641-1647.

13. Tillisch K, Labus JS. Advances in imaging the brain-gut axis: functional gastrointestinal disorders. Gastroenterology 2011;140:407411.e1

14. Pezawas L, Meyer-Lindenberg A, Drabant EM, et al. 5-HTTLPR polymorphism impacts human cingulate-amygdala interactions: a genetic susceptibility mechanism for depression. Nat Neurosci 2005;8: 828-834. 